\title{
Tratamientos empleados en el Instituto
}

\author{
Por el Profesor JOSE DEL CARMEN ACOSTA V.
}

Como se ha visto por los relatos anteriores, en el Instituto se han empleado numerosos tratamientos en los casos de Toxemia y con el transcurso del tiempo y mediante la experiencia adquirida, se han ido precisando los conceptos y fijando las indicaciones de cada uno de ellos; en general, en lo cual estamos acordes con los principios aceptados hoy universalmente, se tiende a estabilizar y extender el tratamien: médico, reduciendo las intervenciones obstétricas y quirúrgicas a indicacione uy limitadas en los casos puramente tóxicos.

Como para toda terapéutica, la base de la indicación es el estudio clínico minucoso del caso para saber de qué forma de la toxicosis se trata y en qué condiciones te halla.

Partiendo de la división en formas con retención de agua y formas secas; consideraremos en primer lugar el tratamiento de las primeras, para el cual en términos generales, desde hace muchos años. hemos adoptado el tratamiento aconsejado por Arnold y Fay, que se basa en la antigua teoría de Zangenmeister.

Según ellos la causa determinante del cuadro clínico sería la retención de agua, que para que tenga lugar requiere la retención previa del ión sodio; aparte de los $\epsilon$ mas caracteriza esta forma de la afección, la elevación tensional que en ocasiopasa de 20 centímetros en la máxima. pero que conserva siempre una amplia rencial entre la máxima y la minima. El tratamiento, que es absolutamente ló-

o desde luego que va a combatir la eausa misma de la afección según ellos, consiste en disminuír o suprimir la causa determinante de los edemas, provocar la eliminación del agua retenida y bajar la tension arterial.

Como primera medida debe establecerse el balance de los líquidos ingeridos y eliminados por la paciente para dejar siempre un margen favorable a la eliminación, de ahí que se recomiende un periodo de dieta con supresión casi completa de liquido durante 24 horas para observar y medir la cantidad eliminada espontáneamente y a la vez hacer la dosificación de la albuminuria y la investigación del sedimento urnario.

En vista de que la filtración renal está muy disminuida, debido al edema y espasmos vascular del parenquima renal, se busca la derivación de la eliminación de los liquidos a través de la mucosa intestinal; con este fin se administra una solución saturada de sulfato de magnesia, en forma de cucharadas, o de copas repetidas frecuentemente, hasta obtener, no un simple efecto purgante, sino una verdadera dia- 
rrea serosa, "sangría bianca": la administración de esta solución en la concentración dicha tiene por objeto dar el máximun de sal de magnesio, con el minimun de agua ya que los tejidos retienen aún el agua de los medicamentos; por otra parte, la gran concentración saliná dentro de la luz intestinal favorece el establecimiento de una abundantisima corriente liquida de los vasos hacia el intestino y finalmente el magnesio abscrbido obra como sedante del sistema nervioso central y por consiguiente disminuye sj imitabilidad y la aparición de las crisis convulsivas. Esta derivacion del agua contenida en el aparato vascular disminuye la plétora sanguínea y por consiguiente baja la tensión arterial, descenso que es coadyuvado por la acción sedarte del magresio.

Es necesario luégo movilizar el agua retenida en los espacios intercelulares $\mathrm{y}$ llevarla a la luz de los vasos, para que por ellos vayan a buscar su puerta de salida en el intestino; con exte fin se administra por vía intravenosa una solución de glucosa al $50 \%$ en cantidad de 40 a 59 centímetros cubicos por dosis, que se repite con intervaics de 4 a 6 horas; debe advertirse que no es indiferente el título de la solución. Ya que lo que se busca es producir un aumento notorio de la tensión osmótica intravascular para que se establezca la corriente de agua de los tejidos hacia el interior de los vasos; de exta manera se cisminuye el edema del riñon y se restablece su funcionamiento con aumento notorio en la eliminación urinaria. EI régimen alimenticio en estas condiciones debe hacerse con supresión completa de de toda adicion de sal de sodio, sin perjuicio del suministro de proteinas y vigilando siempre $\epsilon$ balance de los liquidos; hasta aquí el tratamiento empleado en los casos de albuminurias y preeclampsias leves, complementado naturalmente con el reposo en lá came y el abrigo adecuado de la paciente.

En lo. casos de precelampsias graves se complementa el tratamiento con la administración de un barbitúrico, de preferencia Luminal o Gardenal Sódico por inyeccion intramuscular, a la dosis de 20 a 25 centígramos, repetida con la frecuencia que sea necesaria para mantener tranquila a la enferma. Cuando las crisis convulsivas. ya han aparecido y no cecien al tratamiento aplicado hasta aquí, se añade la punción lumbar, con la extracción de 30 a 40 centímetros cúbicos de líquido cefalorraquideo, para disminuir la tensión intracraneana y la irritación de la corteza cerebral determinante de las convulsiones.

Cuando a pesár de la reducción o desaparición de los edemas, subsiste la hi pertensión y algunas olras manifestaciones tóxicas, es necesario pensar en la probabilidad de la existencia de lesiones renales anteriores al embarazo o secundarias a él, pero que en todo caso requieren un tratamiento diferente.

Cuando se trata de formas sin retención hidrica, formas que llamamos secas: sería ilogico el aplicar el tratamiento descrito, ya que no hay reiención de agua. y por consiguiente no tendría razón de ser para la aplicación; en tales casos, viene empleándose (l) tratameinto a base de veratrum viride, preconizado hace 20 años por la Escucla de Cincinati, producto con el cual se obtiene los mayores y más rá pidos descensos de la tensión arterial.

El plan general de su aplicación puede resumirse así:

1.-Inyección intramuscular profunda de extracto flúido de veratrum viride en dosis de scis décimos de centímetro cúbico $(0,6 \mathrm{cc}$. $)$.

2 -Inyección intramuscular profunda de cinco centimetros cúbicos ( 5 c.c.) de solución de sulfato de magnesia al $10 \%$.

$3^{0}$-Inyección intravenosa de solución de dextrosa o glucosa al $10 \%$, en doge de mil a mil quinientos centímetros cúbicos ( 1.000 a 1.500 c.c.). 
Medida la tensión arterial de la paciente se le aplica la primera dosis de veratrum y se continúa la vigilancia de la tensión, la que en el curso de 20 a $30 \mathrm{mi}-$ nutos empieza a descender de manera progresiva, hasta llegar en muchas ocasiones a bajar en 80 o más milímetros de mercurio en el curso de tres a cuatro horas; a la vez que baja la tensión el pulso se retarda llegando en muchas ocasiones hasta cuarenta o cuarenta y cinco por minuto, la respiración se hace más lenta y pro. funda; $b$ en ocasiones aparecen vómito y sudor, excepcionalmente diarrea, con la dosis indicada.

Tan pronto como desciende la cifra tersional, se instala la infusión intravenosa de la solución dextrosada y se aplica la inyección de sulfato de magnesia.

La guía para la repetición de la dosis de veratrum, que en ningún caso debe pasar de seis décimas de centímetro, pero que puede reducirse a $4-3$ ó 2 según la necesidad, es por una parte la tensión arterial, que debe mantenerse entre 13 y 15 centimetros la máxima o por debajo de esta cifra; y la frecuencia del pulso, que debe mantenerse entre 60 y 70 como máxima. En casos que una y otro tiendan a subir se repite la aplicación, en la cantidad que se juzgue necesario para evitar los ascensos, de modo que los intervalos entre unas y otras varia con las indicaciones clinicas.

El sulfato de magnesia debe repetirse a intervalos de cuatro a seis horas (4 a 6 horas) y la administración de glucosa puede llegar a 3.000 centímetros en las 24 horas. Debe vigilarse cuiaadosamente la tensión durante la administración de la -lucosa intravenosa para evitar que pueda ocurrir un ascenso.

En los casos en que la paciente tenga mucha agitación. no hay inconveniente en nplear los barbitúricos.

Como el veratrum es un producto muy activo y de alta toxicidad debe manejarse personalmente por los médicos o por una persona debidamente adiestrada en este tratamiento y debe tenerse presente que sus antídotos son la atropina y la morfina, que hasta ahora no han tenido que ser empleados en ninguno de los casos tratados en el Instituto.

Con resultados iguales se ha empleado la Veratrone, en los casos en que se nos a agotado el extracto flúido de Veratrum, usando dosis más altas de acuerdo con la lución a que corresponda el producto empleado.

Estos han sido los tratamientos que con mayor frecuencia se han empleado, pero lado de ellos también se han aplicado las jnyecciones intravenosas de Novocaina a. 1 r en dosis de 10 a 15 centímetros, repetidas a intervalos de cuatro a seis hor 2 , que determinan descensos tensionales menos notorios y de duración más corta, pero cue tiene una acción hipnótica y sedante muy benéfica. También se han empleado, aunque hasta ahora en muy pequeño número de casos, los antihistamínicos y la anestesia raquidea. 